\title{
The importance of pial blood supply to the development of peritumoral brain edema in meningiomas
}

Michael Bitzer, M.D., Lars Wöckel, M.D., Andreas R. Luft, Ajay K. Wakhloo, M.D., Ph.D., Dirk Petersen, M.D., Holger Opitz, M.D., Theo Sievert, M.D., Ulrike Ernemann, M.D., and Karsten Voigt, M.D.

Departments of Neuroradiology and Neurosurgery, and the Institute for Brain Research, University of Tübingen, Germany; and Department of Neurosurgery, State University of New York at Buffalo, Buffalo, New York

The authors studied the pial and dural blood supplies in 74 intracranial meningiomas and quantified their associated peritumoral brain edema (PTBE). The extent and localization of pial blush in relation to the total tumor volume were determined angiographically. The amount of edema and tumor size were calculated using computerized tomography. The edema-tumor volume ratio was defined as Edema Index (EI). There were 49 meningiomas with PTBE; of those tumors, 46 were supplied by pial vessels, and three were supplied exclusively by dural vessels. Tumors without PTBE showed no pial blush. The mean EI in meningiomas with pial blush was significantly larger $(E I=3.0)$ than in meningiomas without pial supply $(\mathrm{EI}=1.1 ; \mathrm{p}<0.0001)$. Meningiomas in which $10 \%$ of the whole tumor volume was supplied by pial vessels had only a small mean EI of 2.2, whereas tumors with pial blood supply greater than or equal to $20 \%$ had a mean EI of 3.3 ( $\mathrm{p}<0.026$ ). In $69.9 \%$ of cases with pial blood supply, major portions of the edema were located adjacent to the tumor region supplied by pial vessels. Edema index differences among tumors of different subgroups, as defined by size or histology, were significantly related to the pial supply in each subset. Thus, pial blood supply may be causative for the development of PTBE in meningiomas.

Key Words * meningioma * pial blush * brain edema * vascular supply

Approximately $60 \%$ of meningiomas are associated with a perifocal edema,[4,21,26] which may correlate to tumor size.[11,28] The exact pathophysiological mechanism of cerebral edema formation in meningioma remains unclear. Various causative factors have been discussed including the tumor size, location of the tumor, and histological differentiation. $[4,11,15]$ Previous studies using electron microscopy have described the vasogenic nature of peritumoral brain edema (PTBE) in meningioma.[10] Thus, an impairment of the blood-brain barrier as a primary causative factor seems likely,[13] although meningiomas grow primarily extraaxially. Philippon and colleagues[22] postulated a secretory activity of the tumor. Other authors speculated that brain ischemia[30] or a compromised physiological venous drainage[12] resulting from tumor mass effects might be the cause of PTBE. Edema may also be related to hormone receptors, which have been found in meningioma cells.[2] Inamura, et al.,[15] proposed that the type of arterial blood supply to the tumor is a causative factor for peritumoral edema. 
We investigated the relationship among arterial blood supply, the influences of tumor size and histology, and PTBE in 74 intracranial meningiomas.

\section{CLINICAL MATERIAL AND METHODS}

\section{Patient Selection}

Between 1986 and 1995, 74 patients were examined ( 24 men, 50 women; age range 30-78 years, mean 56.9 years) harboring 74 primary intracranial meningiomas at different locations (Table 1).

Computerized tomography (CT) and angiography studies were obtained in all cases. All patients underwent microsurgical removal of their tumor at our facility. Surgically resected meningiomas were classified histopathologically based on the criteria of the new World Health Organization (WHO) classification of tumors of the central nervous system.[16,17,19,20] Fifty-seven tumors were graded as typical meningiomas (Grade I), five as atypical (Grade II), and 12 as anaplastic meningiomas (Grade III).

\begin{tabular}{|cc|}
\hline \multicolumn{2}{c|}{ TABLE 1 } \\
LOCATION OF PR MARY MEN NGIOMAS IN 74 PATIENTS \\
\hline \hline Location of Weningioma & No. of Patierts \\
\hline convexity & 28 \\
fal $x$ & 18 \\
Sphenoid wing & 6 \\
tem porotasal & 3 \\
tentorial & 2 \\
in fratentorial & 13 \\
other & 4 \\
total & 74 \\
\hline
\end{tabular}

Cerebral angiography was performed using either the manual subtraction conventional technique or using the digital subtraction technique. Selective angiography of the common carotid artery, the internal and external carotid arteries, and the vertebral artery was performed. The angiograms provided a safe and accurate differentiation between pial and dural blood supply. Definitive differentiation was possible when superimposition overlap of pial and dural supply was not detectable on vertebral or internal carotid angiograms. Superimposition of vessels was frequently observed on internal carotid artery injections of frontobasal meningiomas. These cases were excluded from the study. Contrast- or noncontrast-enhanced CT scans were acquired in all patients.

\section{Evaluation of Angiography and Computerized Tomography}

The ratio of pial blush to tumor volume was approximated by evaluating the area of pial blush in anteroposterior and lateral angiograms. The extent of pial supply was rated on a 100-point scale, in which 0 was no pial blush and 100 was exclusively pial supply. Measurements were obtained in 10-point increments. 


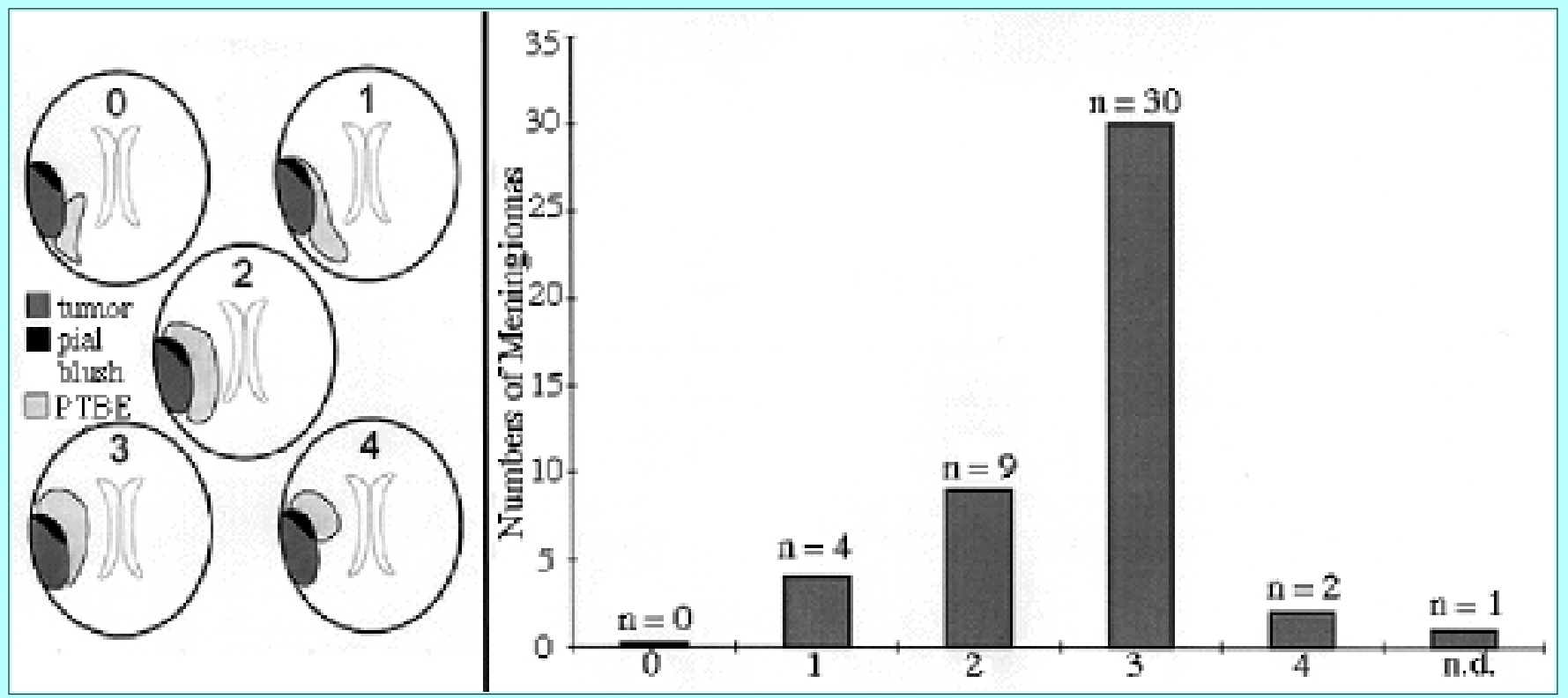

Fig. 1. Graph displaying the spatial relation between pial tumor blush and location of edema in the region of the meningioma. Left: $0=$ edema present but distant from pial blush; $1=$ some edema adjacent to pial blush, but most of edema being far from pial blush; 2 = edema surrounding the tumor in a regular fashion; $3=$ maximum amount of edema adjacent to pial blush; 4 = edema only adjacent to pial blush. Right: Incidence of meningiomas in Groups 0 to 4. n.d. = not definable.

The three-dimensional localization of the pial blush in relation to the tumor surface was obtained from angiograms. The location and center of edema in relation to the tumor surface was estimated from the CT scans. The spatial relationship of both was rated on a scale from 0 to 4 (Fig. 1 left). Tumor and edema volumes were approximated from the CT scans: the maximum perpendicular diameters $(a, b)$ were measured on the axial images and the extent, in coronal direction (c), was estimated from the number of axial images displaying the structure multiplied by the slice thickness. The resulting volume of tumor and edema was then approximated using the formula for a spheroid:

$$
\left(r=\frac{4}{3} J 7 \times\right. \text { ar }
$$

The relation of PTBE and tumor volume, edema index (EI), was defined as

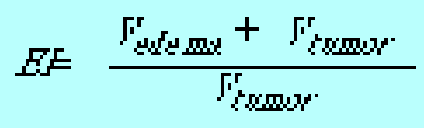

resulting in 1 , when no edema is present.

\section{Statistical Analysis}

Edema indices and frequency of pial blush were statistically evaluated using the Wilcoxon test and the Fisher's exact test (chi-square test), respectively. All calculations were performed using a statistical analysis system (SAS System; SAS Institute Inc., Cary NC). 


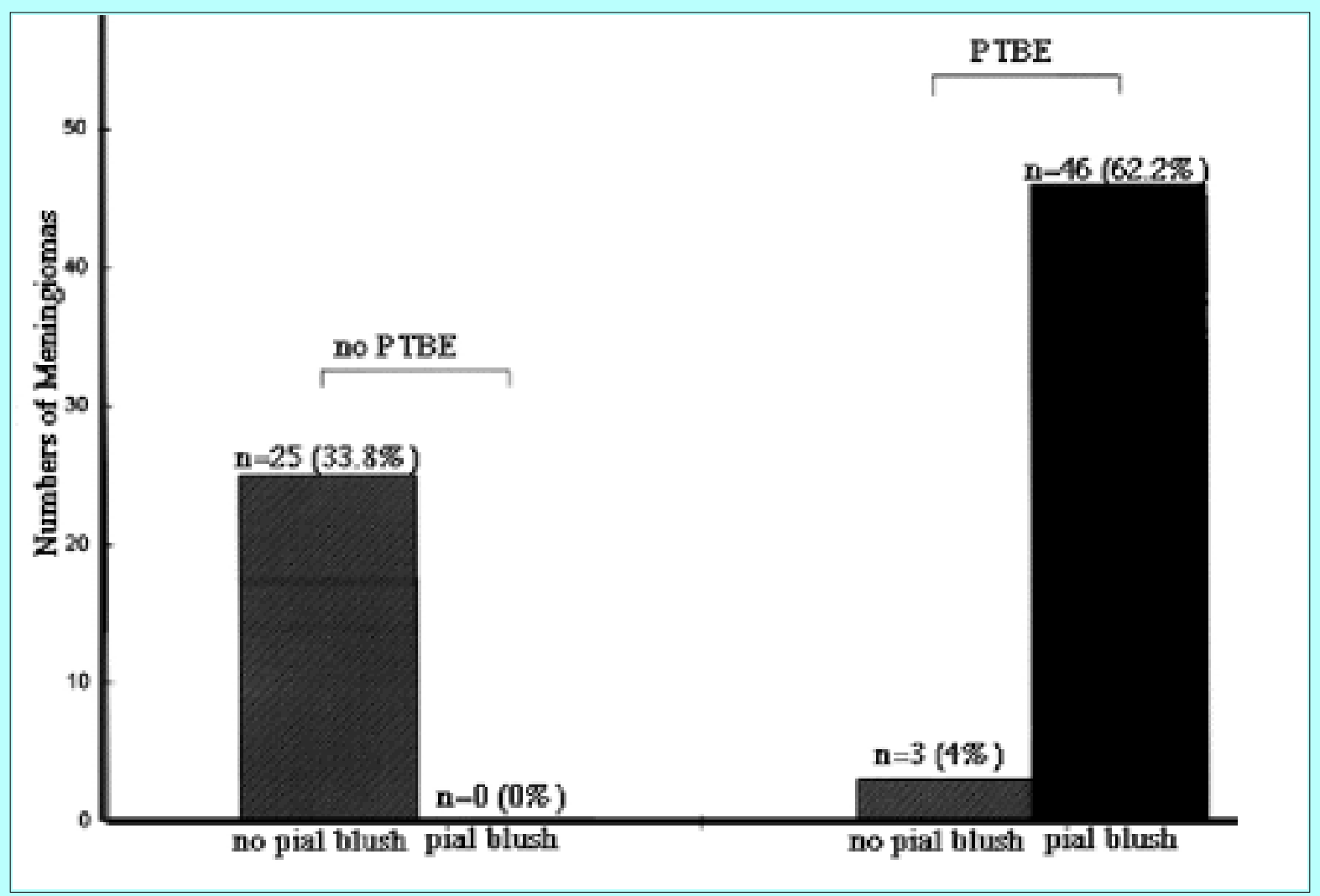

Fig. 2. Graph displaying distribution of frequency of meningiomas related to the presence of edema and pial blush.

\section{RESULTS}

\section{Peritumoral Brain Edema and Vascular Tumor Supply}

Forty-nine of 74 meningiomas demonstrated PTBE on CT scans. The presence of pial blush and PTBE was highly concordant (96\%) (Fig. 2), and only three cases (4\%) presented with PTBE without any detectable pial blush. All of the cases with tumor blood supply through cerebral arteries were associated with edema. 


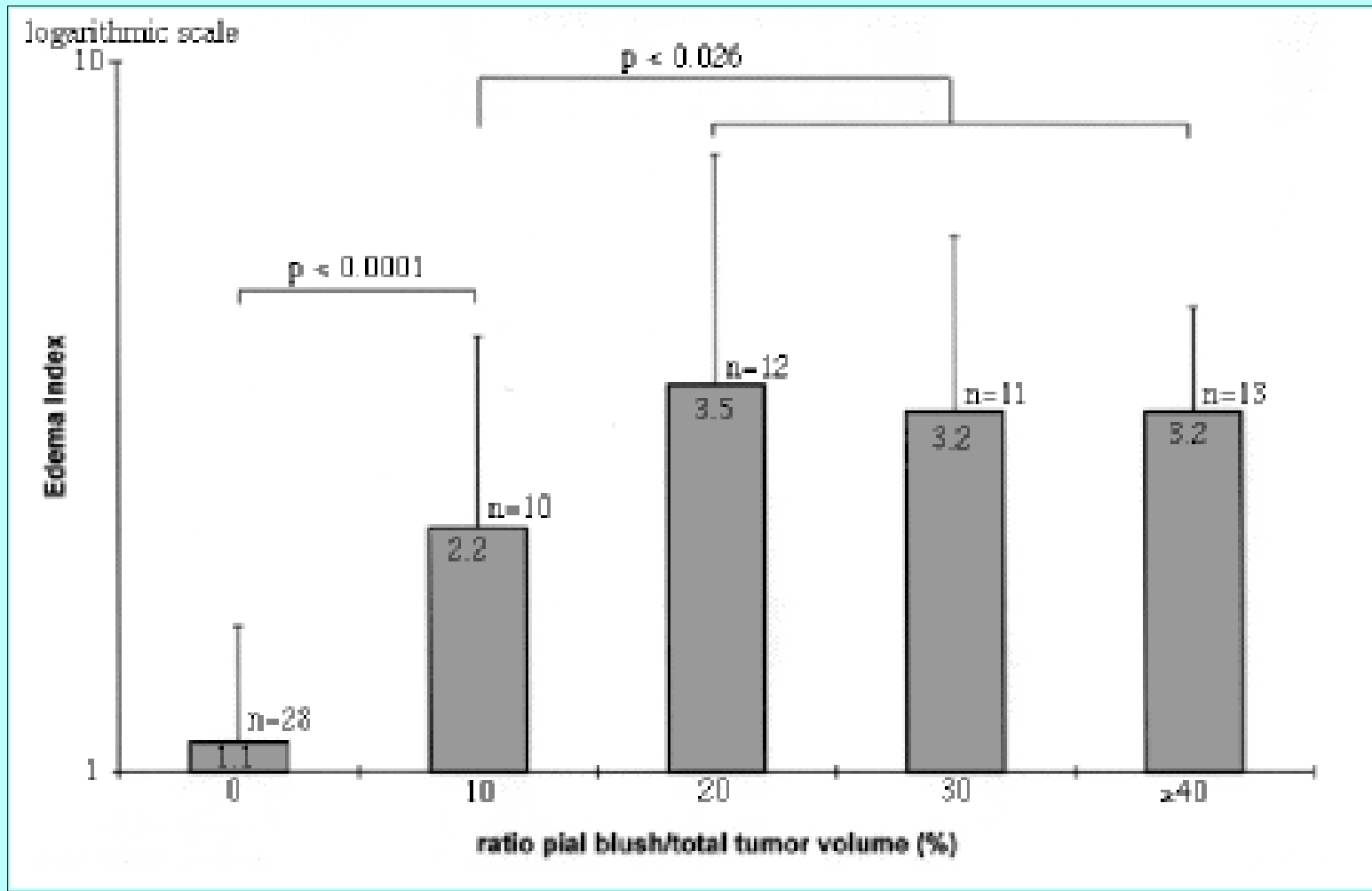

Fig. 3. Graph displaying correlation between EI and the percentage of pially supplied tumor volume.

The EI values, that is, the ratio of edema size to tumor volume were higher in cases with predominantly pial supply (Fig. 3). Meningiomas without pial blush had a small mean EI of 1.1. A minimal increase of pial blush to $10 \%$ of the total tumor volume resulted in an average increase of the mean EI to 2.2 (p < 0.0001 ). A further increase in pial blush to greater than or equal to $20 \%$ of total tumor volume produced a mean EI of 3.3 ( $p<0.026)$. An EI above $20 \%$ showed no further positive correlation with pial blush.

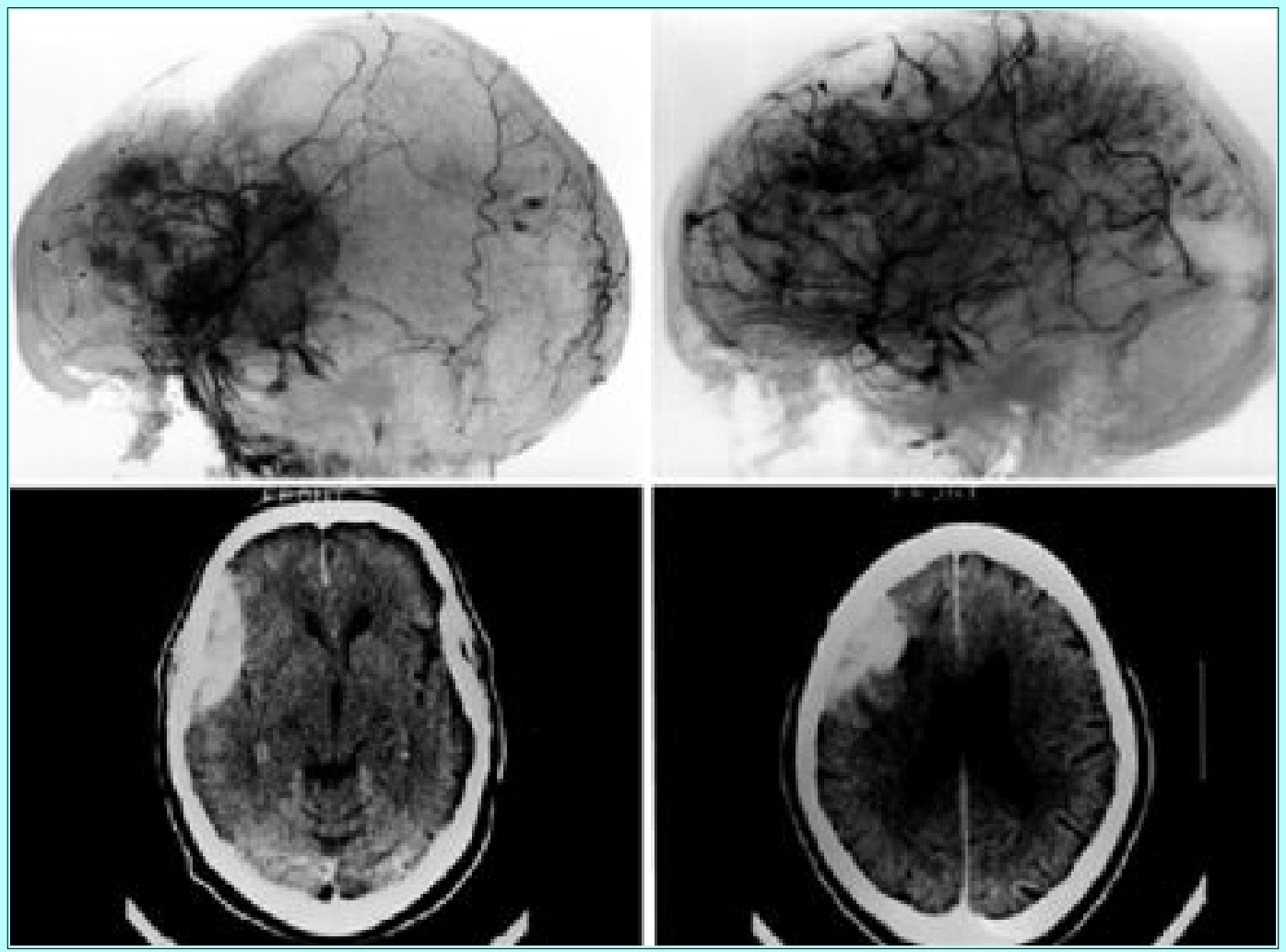


Fig. 4. Large meningioma of the convexity with a small area of PTBE. Upper left: Lateral angiogram of the external carotid artery demonstrating major tumor supply through dural arteries (small arrowheads). Upper right: Lateral angiogram of the left internal carotid artery showing pial supply from cerebral arteries located at the upper apex of the tumor (large arrowheads). Lower left: Computerized tomography scan demonstrating no edema adjacent to the (caudal) region of the tumor where dural blood supply prevails. Lower right:

Computerized tomography scan in the same patient revealing a small edema around the region of pially supplied part of the tumor (arrow).

Notably, the tumor portions supplied through pial vessels were adjacent to the PTBE in $69.6 \%$ of the 46 meningiomas with pial blush (Fig. 1, Fig. 4). In 19.6\% of cases, the edema was either homogeneously distributed around the tumor or followed anatomical borders, whereas in only $8.7 \%$ of cases PTBE and pial areas were scattered.

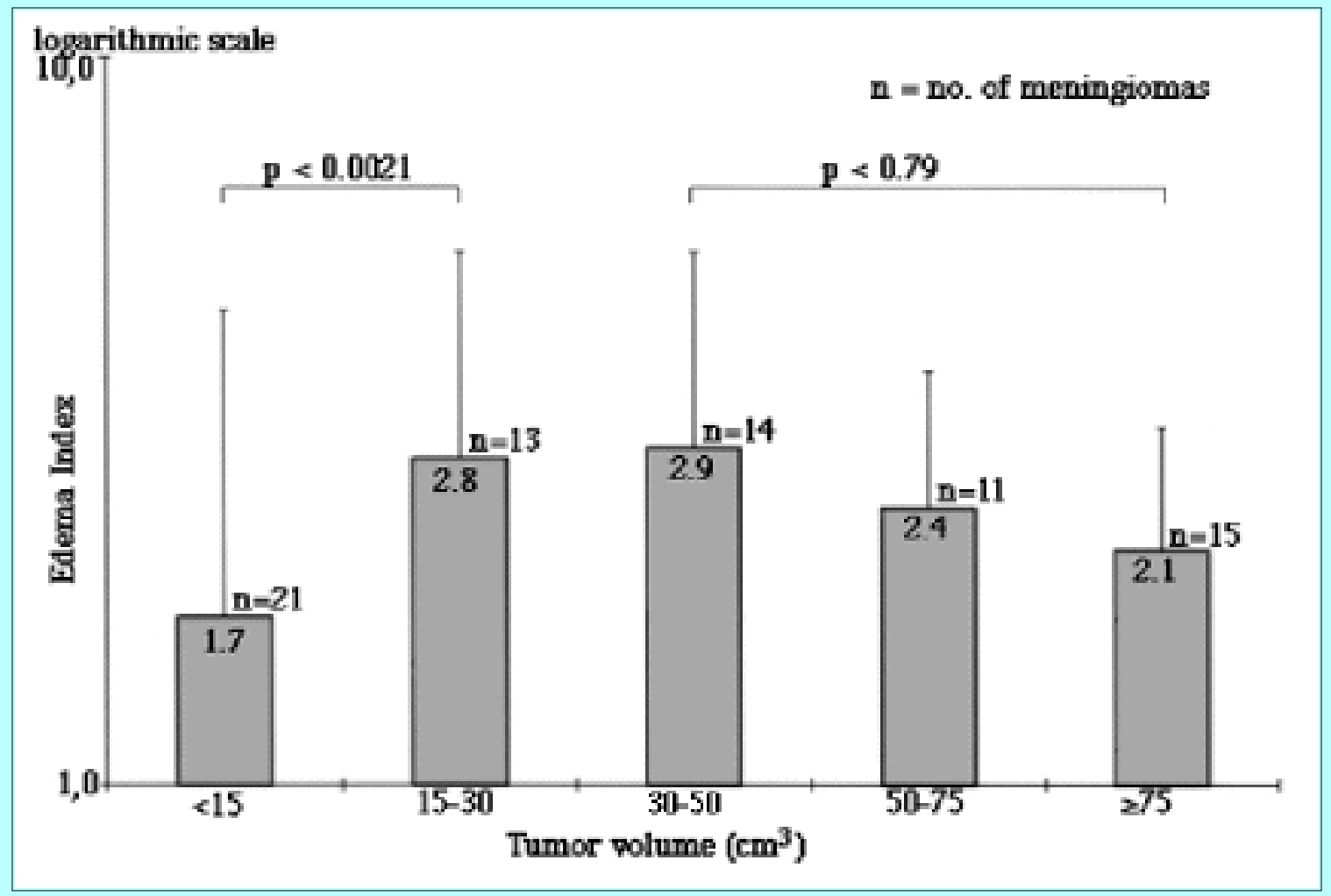

Fig. 5. Graph depicting the correlation of EI and tumor volume.

\section{Tumor Size and Peritumoral Brain Edema}

For small tumors $\left(<15 \mathrm{~cm}^{3}\right)$ the mean EI was significantly smaller than for midsized tumors $\left(15-30 \mathrm{~cm}^{3}\right)$ (Fig. 5). The mean EI was smaller than expected, because the majority of small meningiomas had no detectable edema. However, four of 21 small tumors presented with edema (mean EI =4.9). Two of these cases had a significantly large EI greater than 2; maximum EI $=13.85$; both of these meningiomas were associated with pial blush. On the other hand, two of 15 large tumors $\left(>75 \mathrm{~cm}^{3}\right)$ had no edema and these meningiomas did not reveal any pial blush. Larger tumors showed a simultaneous increase in frequency of edema and pial blush (Fig. 6). 


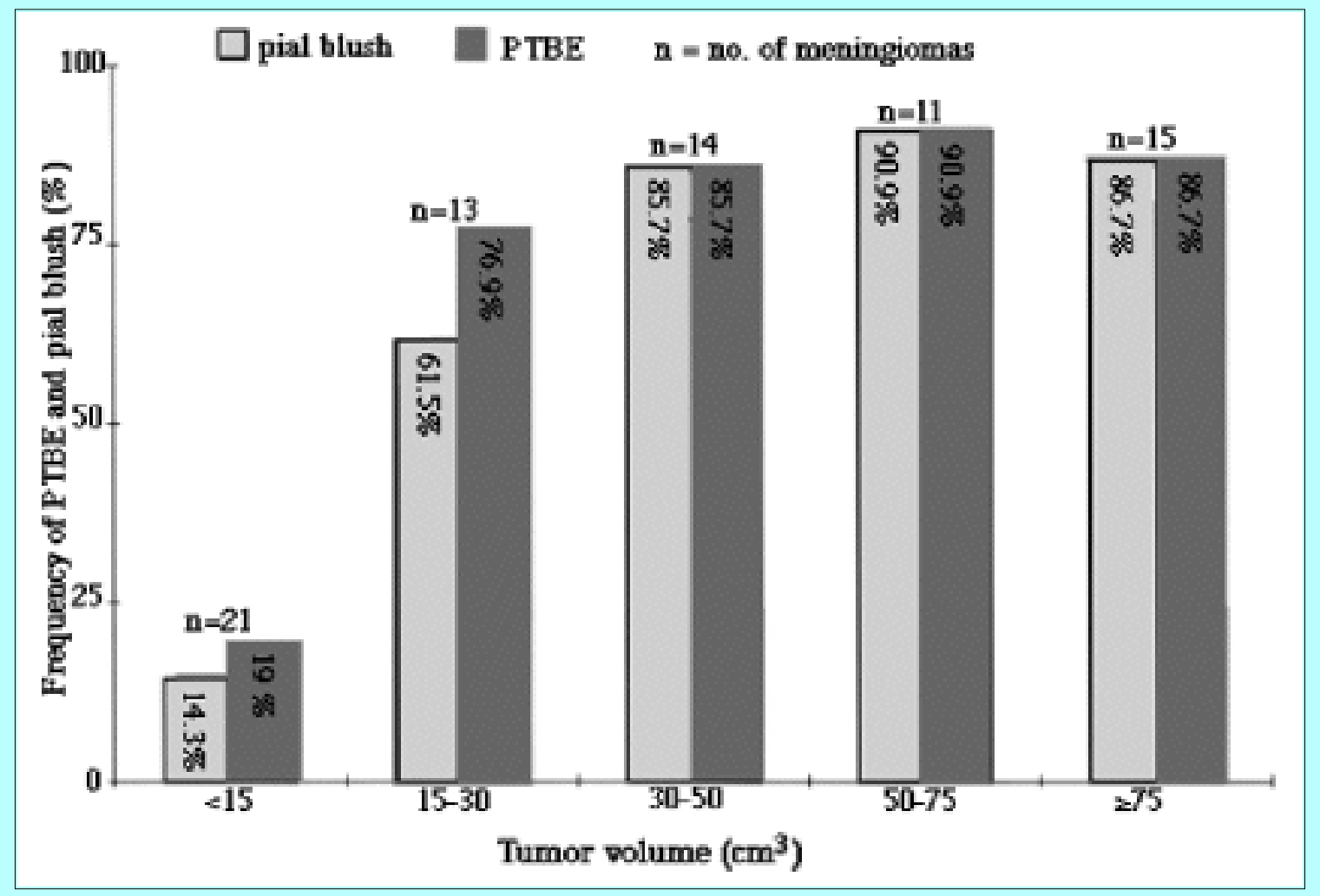

Fig. 6. Graph showing the incidence of PTBE and pial blush in relation to total tumor volume.

\section{Tumor Histology and Peritumoral Brain Edema}

The mean EI correlated with WHO Grades I to III as well as with the subsets of WHO Grade I tumors (meningotheliomatous, transitional, fibroblastic, other). We found a significantly different $\mathrm{EI}(\mathrm{p}<0.007$ ) in WHO Grade I (mean EI = 1.9) and III (mean EI = 3.4) meningiomas. Because of the small number of cases (only five), WHO Grade II tumors could not be sufficiently evaluated. The mean EIs of transitional (2.7) and fibroblastic (1.3) tumors in the WHO Grade I group were significantly different $(\mathrm{p}<0.04)$. 


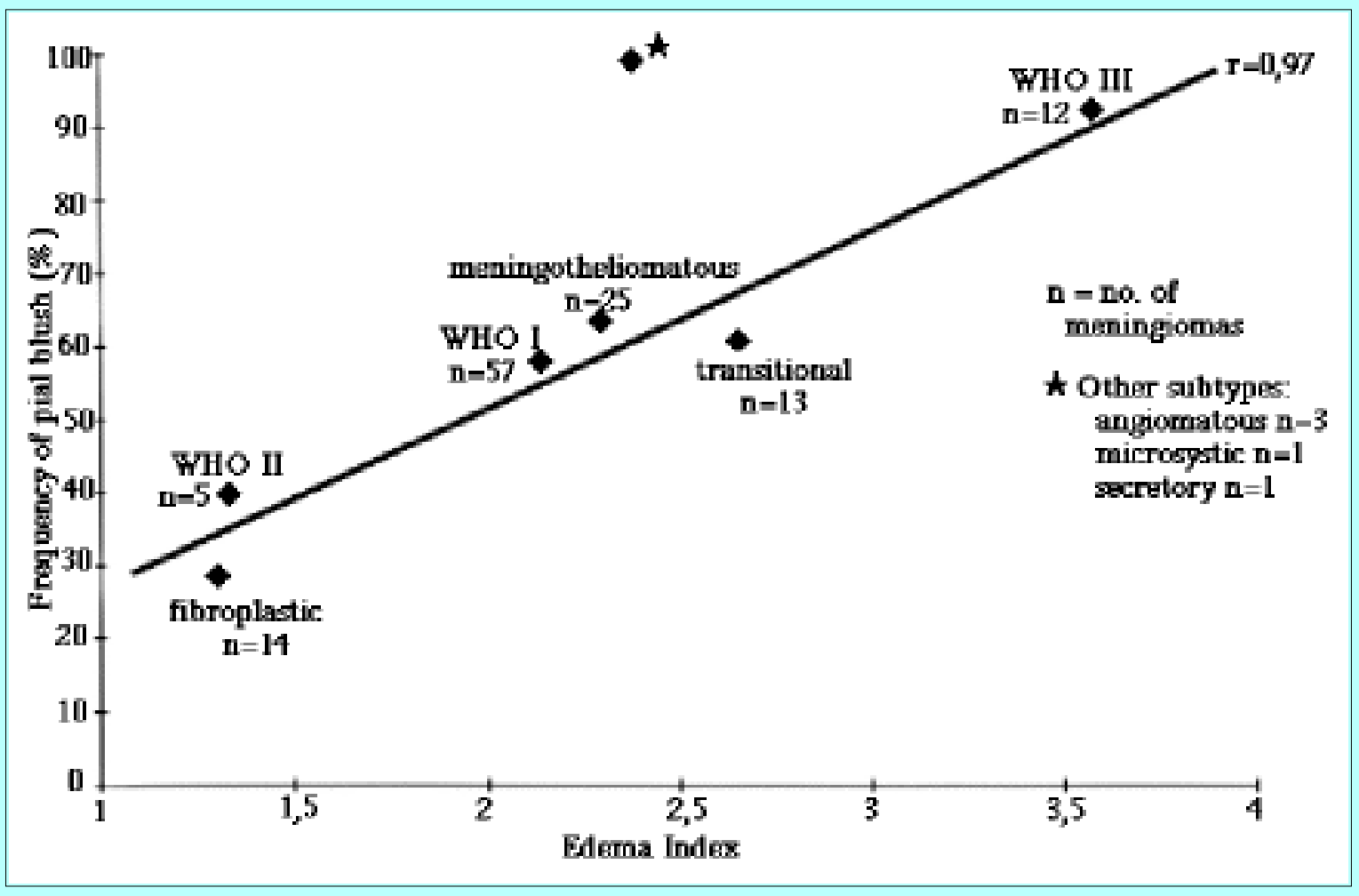

Fig. 7. Graph showing the correlation of EI and incidence of pial blush in relation to histological findings in the tumor. Linear regression was computed for three subgroups of WHO Grade I (meningotheliomatous, transitional, and fibroblastic), WHO Grade II, and III meningiomas. Other subtypes were excluded because of the small number of cases.

The tumor pial blush and the EI correlated within groups of tumors in the same WHO grade. The WHO Grade III tumors were more frequently associated with pial blush $(91.7 \%)$ than the WHO Grade I tumors (57.9\%; $\mathrm{p}<0.05)$. In different histological subtypes, the average EI and the incidence of pial blush showed a high correlation with subtypes $(\mathrm{r}=0.97$; Fig. 7). Six of 12 WHO Grade III tumors with both edema and pial blush had infiltrated the brain parenchyma.

\section{DISCUSSION}

In the present study, we found a qualitative and quantitative correlation between pial blush and PTBE in meningiomas, as evaluated by CT and angiography studies.

The exact pathogenesis of meningioma-associated brain edema is still unknown. However, currently it is widely accepted that the cause of edema is vasogenic rather than cytotoxic. Electron microscopic investigations have revealed similarities between meningioma-associated edema and the experimentally induced vasogenic edema of gliomas.[10] The vasogenic edema in gliomas, as well as cerebral metastases, is related to a disruption of the blood-brain barrier, which permits edema-inducing macromolecules to enter the white matter. Vasogenic edema is more difficult to conceptualize, because meningiomas grow primarily in extracerebral spaces, separated from the brain tissue by the arachnoid, the subarachnoid space, and the pia.

Our study shows that "intrinsic" cerebral arteries may play a significant role in the pathogenesis of meningioma-associated edema. Pial blush in the tumor strongly correlated with the evolution of the edema. This line of reasoning is supported by a case report that demonstrated PTBE in a bifrontal meningioma located adjacent to the area of pial blush.[1] Inamura and colleagues[15] recently reported 
that EI in meningiomas correlated with the occurrence of pial vascularization.

The present study demonstrates that PTBE is significantly related to the degree of pial blood supply and location of pial blush. Although meningiomas displaying small pial blush can induce an edema, the PTBE tends to be smaller compared to tumors with a larger pial supply. Thus, the development of pial supply in meningiomas may be an important step in the genesis of tumor edema. We found a significant correlation between the center of pial blush at the tumor surface and the location of the perifocal edema. This suggests that the edema is either expressed or generated at the tumor surface with high pial supply. The findings of Salpietro and colleagues[24] support this concept. They noted intraoperatively a high incidence of vessels crossing the brain-tumor interface in meningiomas associated with PTBE.

The controversy about the importance of tumor size[5,6,10,11,15,21,24] and histology[4-7,9-11,15,23,25,27] for the genesis of edema has been debated. Our results show that high grade meningiomas (WHO Grade III) with large perifocal edema are also frequently associated with pial supply. On the other hand, tumors with a small amount of edema, such as fibromatous meningioma, are only rarely associated with pial blood supply. Two types of tumors are of particular interest for their pathophysiology: small tumors with a large amount of edema and large tumors with no edema. The lack of pial blood supply in large tumors and the prevailing pial supply in small tumors, in our group of patients, may be the explanation for such extreme cases.

The pial supply of meningiomas reflects the close spatial relationship between the tumor surface and the adjacent brain parenchyma. The arachnoid, which serves as a physiological barrier between the brain and extraaxial structures such as the meningioma, is either penetrated by the cerebral vessels or infiltrated by the tumor; this is similar to some of our WHO Grade III cases. A recently published study demonstrated a strong correlation between the adherence of the tumor to the surrounding brain tissue and the occurrence of edema.[14] Those adherent regions of the tumor showed a disruption of the arachnoid. An intact leptomeninx facilitates only a minimal passage of edema-inducing macromolecules. However, in meningioma tissue the vascular permeability is significantly increased as shown by fluorescence and electron microscopy.[18] Macromolecules enter the interstitium of the meningioma through open gap junctions and fenestrations in the endothelium of capillaries. If the continuity of pia and arachnoid are also impaired, edema-inducing substances and macromolecules may enter the brain tissue.

Angiogeneic factors may be important in the development of blood supply to meningiomas. Vascular endothelial growth factor permeability factor effects the vascularization of meningiomas and other tumors.[3] Moreover, PTBE seems to correlate with the expression of this factor in gliomas as well as in meningiomas.[3,29] Dvorak and coworkers[8] found that vascular endothelial growth factor permeability factor is synthesized in tumor cells and accumulates in the endothelium of tumor vessels. Secretion of these angiogeneic factors into tumor-surrounding region may explain the development of pial blood supply. In addition, these factors may increase the permeability of cerebral vessels, thus facilitating the diffusion of edema-inducing macromolecules into the tumor region.

\section{CONCLUSIONS}

Our study demonstrates the significance of tumor blood supply through pial arteries for the development of PTBE in meningiomas. We found a close spatial relationship between the center of edema and the location of pial blush. Edema size correlated with pial supply in certain subsets of meningiomas that were classified according to their size and histology. 


\section{References}

1. Atkinson JLD, Lane JI: Frontal sagittal meningioma: tumor parasitization of cortical vasculature as the etiology of peritumoral edema. Case Report. J Neurosurg 81:924-926, 1994

2. Benzel EC, Gelder FB: Correlation between sex hormone binding and peritumoral edema in intracranial meningiomas. Neurosurgery 23:169-174, 1988

3. Berkman RA, Merrill MJ, Reinhold WC, et al: Expression of the vascular permeability factor/vascular endothelial growth factor gene in central nervous system neoplasms. J Clin Invest 91:153-159, 1993

4. Bradac GB, Ferszt R, Bender A, et al: Peritumoral edema in meningiomas. A radiological and histological study. Neuroradiology 28:304-312, 1986

5. Constantini S, Tamir J, Gomori MJ, et al: Tumor prostaglandin levels correlate with edema around supratentorial meningiomas. Neurosurgery 33:204-211, 1993

6. De Vries J, Wakhloo AK: Cerebral edema associated with WHO-I, WHO-II, and WHO-III-Meningiomas: correlation of clinical, computed tomographic, operative and histological findings. Acta Neurochir 125:34-40, 1993

7. Dietemann JL, Heldt N, Burguet JL, et al: CT findings in malignant meningiomas. Neuroradiology 23:207-209, 1982

8. Dvorak HF, Sioussat TM, Brown LF, et al: Distribution of vascular permeability factor (vascular endothelial growth factor) in tumors: concentration in tumor blood vessels. J Exp Med 174:1275-1278, 1991

9. Elster AD, Challa VR, Gilbert TH, et al: Meningiomas: MR and histopathologic features. Radiology 170:857-862, 1989

10. Gilbert JJ, Paulseth JE, Coates RK, et al: Cerebral edema associated with meningiomas. Neurosurgery 12:599-605, 1983

11. Go KG, Wilmink JT, Molenaar WM: Peritumoural edema associated with meningiomas. Neurosurgery 23:175-179, 1988

12. Hiyama $\mathrm{H}$, Kubo $\mathrm{O}$, Tajika $\mathrm{Y}$, et al: Meningiomas associated with peritumoural venous stasis: three types on cerebral angiogram. Acta Neurochir 129:31-38, 1994

13. Hossmann KA, Wechsler W, Wilmes F: Experimental peritumorous edema. Morphological and pathophysiological observations. Acta Neuropathol 45:195-203, 1979

14. Ide M, Jimbo M, Kubo O, et al: Peritumoral brain edema and cortical damage by meningioma. Acta Neurochir Suppl 60:369-372, 1994

15. Inamura T, Nishio S, Takeshita I, et al: Peritumoural brain edema in meningiomas: influence of vascular supply on its development. Neurosurgery 31:179-185, 1992

16. Jellinger K, Slowik F: Histological subtypes and prognostic problems in meningiomas. J Neurol 208:279-298, 1975 
17. Kleihues P, Burger PC, Scheithauer BW: Histological Typing of Tumours of the Central Nervous System, ed 2. New York: Springer Verlag, 1993

18. Long DM: Vascular ultrastructure in human meningiomas and schwannomas. J Neurosurg 38:409-419, 1973

19. Maier H, Öfner D, Hittmair A, et al: Classic, atypical, and anaplastic meningioma: three histopathological subtypes of clinical relevance. J Neurosurg 77:616-623, 1992

20. McLean CA, Jolley D, Cukier E, et al: Atypical and malignant meningiomas: importance of micronecrosis as a prognostic indicator. Histopathology 23:349-353, 1993

21. New PF, Aronow S, Hesselink JR: National Cancer Institute study: evaluation of computed tomography in the diagnosis of intracranial neoplasms. IV. Meningiomas. Radiology 136:665-675, 1980 22. Philippon J, Foncin JF, Grob R, et al: Cerebral edema associated with meningiomas: possible role of a secretory-excretory phenomenon. Neurosurgery 14:295-301, 1984

23. Rohringer M, Sutherland GR, Louw DF, et al: Incidence and clinicopathological features of meningioma. J Neurosurg 71:665-672, 1989

24. Salpietro FM, Alafaci C, Lucerna S, et al: Peritumoral edema in meningiomas: microsurgical observations of different tumor interfaces related to computed tomography. Neurosurgery 35:638-642, 1994

25. Servo A, Porras M, Jäskeläinen J, et al: Computed tomography and angiography do not reliably discriminate malignant meningiomas from benign ones. Neuroradiology 32:94-97, 1990

26. Sigel RM, Messina AV: Computed tomography: the anatomic basis of the zone of diminished density surrounding meningiomas. AJR 127:139-141, 1976

27. Smith HP, Challa VR, Moody DM, et al: Biological features of meningiomas that determine the production of cerebral edema. Neurosurgery 8:428-433, 1981

28. Stevens JM, Ruiz JS, Kendall BE: Observations on peritumoral oedema in meningioma. Part II: Mechanisms of oedema production. Neuroradiology 25:125-131, 1983

29. Strugar JG, Criscuolo GR, Rothbart D, et al: Vascular endothelial growth/permeability factor expression in human glioma specimens: correlation with vasogenic brain edema and tumor-associated cysts. J Neurosurg 83:682-689, 1995

30. Tatagiba M, Mirzai S, Samii M: Peritumoral blood flow in intracranial meningiomas. Neurosurgery 28:400-404, 1991

Manuscript received January 28, 1997.

Accepted in final form March 17, 1997.

Address reprint requests to: Michael Bitzer, M.D., Department of Neuroradiology, University of Tübingen, Hoppe-Seyler-Strasse 3, 72076 Tübingen, Germany. 